Pacific Journal of Mathematics

SEPARATION, RETRACTIONS AND HOMOTOPY EXTENSION 


\title{
SEPARATION, RETRACTIONS AND HOMOTOPY EXTENSION IN SEMIALGEBRAIC SPACES
}

\author{
Hans DelfS AND MaNFREd KNebusch
}

\begin{abstract}
We consider an affine semialgebraic space $M$ over a real closed field $R$. Tietze's extension theorem holds in $M$. Every closed semialgebraic subset $A$ of $M$ is a strong deformation retract of a semialgebraic neighbourhood $Z$ in $M$, and $(M, A)$ has the homotopy extension property. If $A$ is locally complete then $Z$ can be chosen as a mapping cylinder.
\end{abstract}

Let $R$ be a real closed field. In [DK $\mathbf{K}_{2}$ ] we developed a theory of semialgebraic spaces and mappings over $R$, and in [D], [DK $\mathbf{D K}_{3}$, we showed that for an affine semialgebraic space $M$ over $R$ there exist reasonable homology and cohomology groups $H_{r}(M, G), H^{r}(M, G)$ with coefficients in an arbitrary abelian group $G$ which coincide with the classical singular homology and cohomology groups in the case $R=\mathbf{R}$. There should also exist a reasonable homotopy theory for such spaces, and this theory - as well as homology theory - should certainly be useful in algebraic geometry over $R$ and over $R(\sqrt{-1})$.

The present paper serves as ground work for semialgebraic homotopy theory. As in topological homotopy theory, we have to make sure, for example, that under sufficiently general assumptions a subspace $A$ of $M$ has a semialgebraic neighbourhood $U$ in $M$ which retracts to $A$, and that the pair $(M, A)$ has the homotopy extension property for semialgebraic maps. It turns out that in the category of affine semialgebraic spaces these matters are even nicer than in topology.

In $\$ 1$ we show that affine semialgebraic spaces have separation properties similar to paracompact spaces, a fact which is particularly useful for the sheaf theory of these spaces (a topic we do not consider here, cf. [D]). $\$ 2$ is devoted to a proof of our central result, Theorem 2.1 below, which in a slightly weaker and simpler form says the following.

THEOREM 1. Any closed semialgebraic subset $A$ of an affine semialgebraic space $M$ has an open semialgebraic neighbourhood $U$ in $M$ such that $A$ is a strong deformation retract of both $U$ and of the closure $\bar{U}$ of $U$ in $M$ (in the semialgebraic sense).

We feel that this result is a remarkable instance of the good-natured behaviour of the affine semialgebraic category over an arbitrary real closed field $R$. 
We prove a rather constructive version of the theorem (Theorem 2.7). We rely heavily on the result that every semialgebraic pair $(M, A)$ with $M$ affine can be "triangulated" ([D], [DK $\left.\left.\mathbf{D K}_{\mathbf{3}}, \S 2\right]\right)$. This triangulation is only of a semiclassical nature (some open simplices in the occuring polytopes are "missing".) Thus arguments have to be used, which, to some extent, seem to be new even in the case $R=\mathbf{R}$.

In $\S 3$ we introduce locally complete semialgebraic spaces. We verify that these spaces are precisely the euclidean neighbourhood retracts in the semialgebraic sense (Theorem 3.6). We also obtain the following improvement of Theorem 1 for locally complete spaces (again in a very constructive version, cf. end of $\S 3$ ).

THEOREM 2. Let $M$ be a locally complete space (cf. Def. 3.1), and $A$ be a closed semialgebraic subspace of $M$. Then there exists an open semialgebraic neighbourhood $U$ of $A$ in $M$ and a proper semialgebraic map $f$ : $\partial U \rightarrow A$, with $\partial U=\bar{U} \backslash U$, such that the triple $(\bar{U}, A, \partial U)$ is semialgebraically isomorphic to the triple $(Z(f), A, \partial U)$ consisting of the mapping cylinder $Z(f)$ of $f$ and the natural subspaces $A$ and $\partial U$ of $Z(f)$.

This result applies, for example, to the case where $M$ is the set $V(R)$ of rational points of any algebraic variety $V$ over $R$, since such spaces $V(R)$ are always locally complete (Example 3.2).

Returning to arbitrary affine semialgebraic spaces we obtain in $\S 4$, among other results, the following consequence of $\S 1$ and Theorem 1.

Theorem 3. ( $C f$. Theorem 4.5 below.) If $M$ is an affine semialgebraic space over $R, A$ a closed semialgebraic subset of $M$ and $N$ a contractible semialgebraic space, then every semialgebraic map $f: A \rightarrow N$ can be extended to a semialgebraic map $\tilde{f}: M \rightarrow N$.

Notice that for $N$ an interval in $R$ this theorem is a semialgebraic analogue of the well known Tietze extension theorem in topology.

We finally prove in $\$ 5$

TheOREM 4. (= Theorem 5.1.) Let I denote the unit interval $[0,1]$ of $R$. Let $M$ be an affine semialgebraic space and $A$ a closed semialgebraic subset of $M$. Then $(A \times I) \cup(M \times\{0\})$ is a strong deformation retract of $M \times I$. 
Thus the pair $(M, A)$ has the homotopy extension property for semialgebraic maps.

The category of affine semialgebraic spaces over the field $\mathbf{R}$ of real numbers, lying “in between' the category PL of piecewise linear spaces and the category TOP of topological spaces, is very convenient, since it often behaves less rigidly than PL and less pathologically than TOP. Thus we hope that our paper - and the whole program pursued here - is also relevant to topologists and geometers only interested in the case $R=\mathbf{R}$. For an outline of this program we refer the reader to $\S 8.13$ of Brumfiel's book [B] and to the introduction of $\left[\mathbf{D K}_{\mathbf{1}}\right]$.

Our methods are restricted to affine semialgebraic spaces. An affine semialgebraic space $M$ over $R$ is just a semialgebraic set $M$ in some $R^{n}$, "regarded without reference to the embedding $M \hookrightarrow R^{n}$ ", cf. [DK $\left.2, \S 7\right]$. According to a recent rather deep result of Robson $[\mathbf{R}]$, cited in $\S 1$ as Theorem 1.3, every semialgebraic space with mild separation properties is affine. Robson's criterion seems to be sufficient to verify that most semialgebraic spaces which are constructed from affine spaces in a reasonable way are affine. Also, the set $V(R)$ of rational points of any separated algebraic variety $V$ over $R$ is affine (cf. Example 3.2 and Proposition 3.5 below). Thus affine spaces seem to suffice for all practical purposes.

We thank the referee for a substantial improvement of the first version of his paper. He provided us with an explicit formula for the retraction in Theorem 1, while previously we obtained the retraction in a rather indirect way. This formula made the proof of Theorem 1 much simpler and enabled us to prove Theorem 2.

\section{Separation properties of affine semialgebraic spaces.}

LEMMA 1.1. Let $A$ be a closed semialgebraic subset of an affine semialgebraic space $M$ over $R$. Then there exists a semialgebraic function $f$ : $M \rightarrow[0,1]$ from $M$ to the unit interval in $R$ such that $f^{-1}(0)=A$.

Proof. We embed $M$ into some $R^{n}$. The distance function $g: R^{n} \rightarrow R$,

$$
g(x):=\min \{\|x-y\| \mid y \in \bar{A}\},
$$

where $\bar{A}$ denotes the closure of $A$ in $R^{n}$, is well defined and semialgebraic $\left(\left[\mathbf{D K}_{2}, 7.8\right],[\mathbf{B}, 8.13 .12]\right)$. Define $f$ as the minimum of $g \mid M$ and the constant function 1. Then $f$ has the required properties. 
Proposition 1.2. Let $M$ be an affine semialgebraic space and let $A, B$ be disjoint closed semialgebraic subsets of $M$. Then there exist open semialgebraic neighbourhoods $U$ of $A$ and $V$ of $B$ with $U \cap V=\varnothing$.

Proof. By the preceding lemma we have semialgebraic functions $f$ and $g$ on $M$ with values in $[0,1]$ such that $f^{-1}(0)=A$ and $g^{-1}(0)=B$. The sets

$$
\begin{aligned}
& U:=\{x \in M \mid(f-g)(x)<0\} \\
& V:=\{x \in M \mid(f-g)(x)>0\}
\end{aligned}
$$

are disjoint, open and semialgebraic, and $U \supset A, V \supset B$.

R. Robson has recently proved a strong converse of this proposition.

THEOREM $1.3[\mathbf{R}]$. Let $M$ be a semialgebraic space over $R$ which is "regular", i.e., if $A$ is a closed semialgebraic subset of $M$ and $x$ is a point in $M \backslash A$, then there exist open semialgebraic neighbourhoods $U$ of $x$ and $V$ of $A$ with $U \cap V=\varnothing$. Then $M$ is affine.

This remarkable result will be used in the present paper only in an inessential way. But it tells us, that the restriction to affine spaces in most of our considerations here is in fact very natural (cf. Introduction).

LEMMA 1.4. (Shrinking of open coverings.) Let $\left(U_{l} \mid i=1,2, \ldots, n\right)$ be a finite covering of an affine semialgebraic space $M$ by open semialgebraic subsets $U_{l}$. Then there exist open semialgebraic subsets $V_{i}$ of $U_{l}, 1 \leq i \leq n$, whose closures $\bar{V}_{l}$ in $M$ are contained in $U_{i}$ and which still cover $M$, i.e. $M=V_{1} \cup \cdots \cup V_{n}$.

Proof. We define $V_{l}$ by induction on $i$. Assume the open sets $V_{l}$ are already defined for $1 \leq i \leq m(0 \leq m \leq n-1)$, such that $\overline{V_{l}} \subset U_{l}$ and

$$
\left(\bigcup_{l=1}^{m} V_{i}\right) \cup\left(\bigcup_{l=m+1}^{n} U_{i}\right)=M
$$

The boundary $\partial U_{m+1}$ of $U_{m+1}$ in $M$ is a closed semialgebraic subset of $M$ as well as

$$
A_{m}:=M \backslash\left[\left(\bigcup_{i=1}^{m} V_{l}\right) \cup\left(\bigcup_{i=m+2}^{n} U_{l}\right)\right] .
$$

The intersection $\left(\partial U_{m+1}\right) \cap A_{m}$ is empty. By Proposition 1.2 there exist open semialgebraic neighbourhoods $T$ of $A_{m}$ and $W$ of $\partial U_{m+1}$ with 
$T \cap W=\varnothing$. We define

$$
V_{m+1}:=T \cap U_{m+1} .
$$

Then $\bar{V}_{m+1} \subset U_{m+1}$ and obviously

$$
\left(\bigcup_{i=1}^{m+1} V_{i}\right) \cup\left(\bigcup_{i=m+2}^{n} U_{i}\right)=M .
$$

Proposition 1.5. (Semialgebraic partition of unity.) Again let $\left(U_{i} \mid i \in\right.$ $I)$ be a finite covering of an affine semialgebraic space $M$ by open semialgebraic sets $U_{i}$. Then there exists a family $\left(f_{i} \mid i \in I\right)$ of semialgebraic functions $f_{i}$ on $M$ with values in $[0,1]$ such that

(a) $\operatorname{supp}\left(f_{i}\right):=\overline{\left\{x \in M \mid f_{i}(x) \neq 0\right\}} \subset U_{i}$ for every $i \in I$.

(b) $\sum_{i \in I} f_{i}(x)=1$ for every $x \in M$.

Proof. We choose a "shrinking" $\left(V_{i} \mid i \in I\right)$ of the covering $\left(U_{i} \mid i \in I\right)$ as described in the preceding lemma. According to Lemma 1.1 we have semialgebraic functions $g_{i}: M \rightarrow[0,1]$ with $g_{i}^{-1}(0)=M \backslash V_{i}$. The functions

$$
f_{i}:=g_{i} \cdot\left[\sum_{j \in I} g_{j}\right]^{-1} \quad(i \in I)
$$

fulfill all the requirements.

We now obtain the following improvement of Proposition 1.2.

THEOREM 1.6. Let $M$ be an affine semialgebraic space over $R$ and let $A$, $B$ be disjoint closed semialgebraic subsets of $M$. Then there exists a semialgebraic function $f: M \rightarrow[0,1]$ with $f^{-1}(0)=A$ and $f^{-1}(1)=B$.

Proof. We choose disjoint open semialgebraic neighbourhoods $U$ of $A$ and $V$ of $B$ (Prop. 1.2), and consider the covering of $M$ by $U, V$, and $W:=M \backslash(A \cup B)$. Let $\left(s_{U}, s_{V}, s_{W}\right)$ be a semialgebraic partition of unity subordinated to this covering, as described in the preceding proposition. Applying again Lemma 1.1 we get a semialgebraic function $g_{U}$ on $M$ with values in $\left[0, \frac{1}{2}\right]$ and $g_{U}^{-1}(0)=A$, and a semialgebraic function $g_{V}$ on $M$ with values in $\left[\frac{1}{2}, 1\right]$ and $g_{V}^{-1}(1)=B$. The function

$$
f:=s_{U} \cdot g_{U}+s_{V} \cdot g_{V}+\frac{1}{2} s_{W}
$$

has the desired properties. 
2. The canonical neighbourhood retraction. We have to recall some terminology from classical homotopy theory, adapted to our semialgebraic setting.

Definition 1. (a) Let $M, N$ be semialgebraic spaces over $R$ and $f, g$ semialgebraic maps from $M$ to $N$. A (semialgebraic) homotopy from $f$ to $g$ is a semialgebraic map $H: M \times[0,1] \rightarrow N$ such that $H_{0}=f$ and $H_{1}=g$. Here $H_{t}$ denotes the map $x \mapsto H(x, t)$ from $M$ to $N(t \in[0,1])$.

(b) A semialgebraic subset $A$ of $M$ is called a retract of $M$ if there exists a semialgebraic map $r: M \rightarrow A$ with $r \mid A=\mathrm{id}_{A}$. Notice that then $A$ must be closed in $M$. Any such map $r$ is called a retraction from $M$ to $A$.

(c) A semialgebraic subset $A$ of $M$ is called a strong deformation retract of $M$, if there exists a homotopy $H: M \times[0,1] \rightarrow M$ such that $H_{0}$ is the identity of $M, H_{1}$ is a retraction from $M$ to $A$ and $H_{t}(a)=a$ for every $a \in A$ and every $t \in[0,1]$. We then call $H$ a strong deformation retraction from $M$ to $A$.

We now start to prove the central result of this paper, namely

THEOREM 2.1. Let $M$ be an affine semialgebraic space and $A$ a closed semialgebraic subset of $M$. Then there exists an open semialgebraic neighbourhood $U$ of $A$ in $M$ and a strong deformation retraction

$$
H: \bar{U} \times[0,1] \rightarrow \bar{U}
$$

from the closure $\bar{U}$ of $U$ in $M$ to $A$, such that also the restriction $H \mid U \times[0,1]$ is a strong deformation retraction from $U$ to $A$.

Actually we shall prove a more precise version of Theorem 2.1 (Theorem 2.7 below). We recall the fact that every pair $(M, A)$ consisting of an affine semialgebraic space $M$ and an arbitrary semialgebraic subspace $A$ can be triangulated, cf. [D, §2] or [ $\mathbf{D K}_{3}$, Th. 2.1]. This means that there exists a geometric simplicial complex $\left(X,\left(S_{i} \mid i \in I\right)\right)$ and a semialgebraic isomorphism $\psi: X \stackrel{\sim}{\rightarrow} M$ such that $\psi^{-1}(A)$ is the underlying set $Y$ of a subcomplex $\left(Y,\left(S_{i} \mid i \in J\right)\right)(J \subset I)$. Here a geometric simplicial complex $\left(X,\left(S_{i} \mid i \in I\right)\right)$ is defined as a subset $X$ of some space $R^{n}$ equipped with a finite partition $\left(S_{i} \mid i \in I\right)$ into (straight) open simplices $S_{l}$, such that the intersection $\bar{S}_{i} \cap \bar{S}_{J}$ of the closures of any two simplices $S_{i}, S_{j}$ in $R^{n}$ is either empty or a face of both $\bar{S}_{t}$ and $\bar{S}_{j}$. Thus the closure $\bar{X}$ of $X$ in $R^{n}$ is obtained from $X$ by adding all open faces of all open simplices $S_{i}$, and $\bar{X}$ is a "finite polyhedron" in the classical sense, called 
here a "complete" geometric simplicial complex. We refer the reader to $\left[\mathbf{D K}_{3}\right.$, §2] for details concerning the terminology we will use from now on in connection with triangulations and geometric simplicial complexes. In addition to this terminology we shall use the following notation. If $e_{0}, \ldots, e_{r}$ are independent points in some $R^{n}$, then $] e_{0}, \ldots, e_{r}$ [ denotes the open simplex and $\left[e_{0}, \ldots, e_{r}\right]$ denotes the closed simplex with vertices $e_{0}, \ldots, e_{r}$. If $S, T$ are open simplices in $R^{n}$ then $S \leq T$ means that $S$ is a face of $T$ and $S<T$ means that $S$ is a proper face of $T$. The barycenter of an open simplex $S$ is denoted by $\hat{S}$.

From now on we will denote a geometric simplicial complex ( $X$, $\left(S_{i} \mid i \in I\right)$ ) simply by the letter $X$ and the set $\left\{S_{i} \mid i \in I\right\}$ of open simplices of $X$ by $\Sigma(X)$.

Definition 2. Let $X$ be a geometric simplicial complex. The star $\mathrm{St}_{X}(A)$ of a semialgebraic subset $A$ of $X$ is the union of all open simplices $S$ of $X$ such that $\bar{S} \cap A \neq \varnothing$. This is clearly an open neighbourhood of $A$ in $X$. If $A$ is a subcomplex of $X$ then $\operatorname{St}_{X}(A)$ is the union of all sets $\mathrm{St}_{X}(T)$ with $T$ running through the open simplices of $A$.

Proposition 2.2. Let $X$ be a geometric simplicial complex and $A$ be a closed subcomplex of $X$. Let $X^{\prime}$ denote the first barycentric subdivision of the complex $X\left(c f .\left[\mathbf{D K}_{3}, \S 2\right.\right.$, Def. 7]). Then there exists a retraction $r$ from the star $V:=\mathrm{St}_{X^{\prime}}(A)$ of $A$ in $X^{\prime}$ to $A$ - to be described explicitly below - with the following property: For every point $x$ in $V$ the open line segment $] x, r(x)[$ is contained in the same open simplex $S \subset V$ of $X^{\prime}$ as $x$.

$N . B$. By this proposition

$$
H(x, t)=(1-t) x+\operatorname{tr}(x) \quad(x \in V, 0 \leq t \leq 1)
$$

is a strong deformation retraction from $V$ to $A$. The image $r(S)$ of every open simplex $S \subset V$ of $X^{\prime}$ is contained in $\bar{S}$.

We need some preparations before we can write down the definition of the retraction $r$. Let $E$ be the set of vertices of $\bar{X}$. Consider a point $x \in \bar{X}$. Then $x$ is contained in an uniquely determined open simplex ]$e_{0}, \ldots, e_{n}[$ of $\bar{X}$,

$$
x=\sum_{l=0}^{n} \lambda_{l} e_{l}, \quad \lambda_{i} \in R, 0<\lambda_{l} \leq 1, \sum_{i=0}^{n} \lambda_{l}=1 .
$$


For $e \in E$ we define

$$
\lambda_{e}(x):= \begin{cases}0 & \text { if } e \notin\left\{e_{0}, \ldots, e_{n}\right\}, \\ \lambda_{i} & \text { if } e=e_{i}(0 \leq i \leq n) .\end{cases}
$$

In this way we get the barycentric coordinate function $\lambda_{e}: \bar{X} \rightarrow[0,1]$. It is semialgebraic. For every $x \in \bar{X}$

$$
\sum_{e \in E} \lambda_{e}(x)=1, \quad x=\sum_{e \in E} \lambda_{e}(x) e,
$$

and $x \in X$ if and only if the vertices $e$ with $\lambda_{e}(x) \neq 0$ span a simplex of $X$.

Now $\{\hat{\sigma} \mid \sigma \in \Sigma(\bar{X})\}$ is the set of vertices of the first barycentric subdivision $\bar{X}^{\prime}$ of $\bar{X}$. Let $\lambda_{\sigma}$ denote the barycentric coordinate function of $\bar{X}^{\prime}$ corresponding to the vertex $\hat{\sigma}$. Thus, for every $x \in \bar{X}$,

$$
\sum_{\sigma \in \Sigma(\bar{X})} \lambda_{\sigma}(x)=1, \quad x=\sum_{\sigma \in \Sigma(\bar{X})} \lambda_{\sigma}(x) \hat{\sigma}
$$

and the vertices $\hat{\sigma}$ with $\lambda_{\sigma}(x) \neq 0$ span the open simplex of $\bar{X}^{\prime}$ which contains $x$. Recall that $\Sigma(\bar{X})$ is partially ordered by the face relation $\sigma<\tau$. By definition of the first barycentric subdivision a subset $\left\{\sigma_{0}, \ldots, \sigma_{n}\right\}$ of $\Sigma(\bar{X})$ is totally ordered if and only if the vertices $\hat{\sigma}_{0}, \ldots, \hat{\sigma}_{n}$ span a simplex of $\bar{X}^{\prime}$. An open simplex $] \hat{\sigma}_{0}, \ldots, \hat{\sigma}_{n}\left[, \sigma_{0}<\cdots<\sigma_{n}\right.$, of $\bar{X}^{\prime}$ is an open simplex of $X^{\prime}$ if and only if $\sigma_{n}$ is contained in $X$.

For every $\sigma \in \Sigma(\bar{X})$ we introduce a function $w_{\sigma}: X \rightarrow[0,1]$ as follows: If $\sigma \subset A$ then $w_{\sigma} \equiv 1$. If $\sigma \subset \bar{X} \backslash \bar{A}$, then $w_{\sigma} \equiv 0$. If $\sigma \subset \bar{A} \backslash A$ then

$$
w_{\sigma}(x)=\left(\sum_{\sigma<\tau \subset X} \lambda_{\tau}(x)\right)^{-1} \cdot\left(\sum_{\sigma<\tau \subset A} \lambda_{\tau}(x)\right),
$$

provided the denominator does not vanish, and $w_{\sigma}(x)=0$ otherwise. (The summations run over all $\tau \in \Sigma(X)$, resp. $\tau \in \Sigma(A)$, with $\sigma<\tau$.) In general, the function $w_{\sigma}$ is not continuous. But the restriction of $w_{\sigma}$ to any open simplex of $X^{\prime}$ is semialgebraic. Thus $w_{\sigma}$ has a semialgebraic graph.

LEMMA 2.3. Let $\sigma$ be an open simplex of $\bar{X}$ with $\sigma \subset \bar{A} \backslash A$. Let $S$ be an open simplex of $X^{\prime}$ on which the function $\Sigma_{\sigma<\tau \subset X} \lambda_{\tau}$ vanishes. Then $\lambda_{\sigma}$ also vanishes on $S$.

Proof. We have $S=] \hat{\sigma}_{0}, \ldots, \hat{\sigma}_{n}$ [ with open simplices $\sigma_{0}<\cdots<\sigma_{n}$ of $\bar{X}$, and $\sigma_{n} \subset X$ since $S \subset X$. Suppose that $\lambda_{\sigma} \neq 0$ somewhere, and hence everywhere, on $S$. Then $\sigma$ coincides with one of the simplices $\sigma_{0}, \ldots, \sigma_{n-1}$. 
(In particular $n \geq 1$ ). Thus

$$
\sum_{\sigma<\tau \subset X} \lambda_{\tau}(x) \geq \lambda_{\sigma_{n}}(x)>0
$$

for every $x \in S$.

Since $0 \leq w_{\sigma}(x) \leq 1$ for every $x \in X$ we see from Lemma 2.3 that the function $\mu_{\sigma}:=w_{\sigma} \cdot \lambda_{\sigma}$ is continuous and hence semialgebraic on $X$ for every $\sigma \in \Sigma(\bar{X})$.

Lemma 2.4. For every $x \in V:=\mathrm{St}_{X^{\prime}}(A)$ there exists some $\sigma \in \Sigma(\bar{X})$ with $\mu_{\sigma}(x)>0$.

Proof. Let $S \subset V$ be an open simplex of $X^{\prime}$. As above, $\left.S=\right] \hat{\sigma}_{0}, \ldots, \hat{\sigma}_{n}[$ with open simplices $\sigma_{0}<\cdots<\sigma_{n}$ of $\bar{X}$ and $\sigma_{n} \subset X$. Since some open face of $S$ is contained in $A$, we have $\sigma_{k} \subset A$ for some $k \in\{0, \ldots, n\}$. For $\sigma=\sigma_{k}$ certainly $\mu_{\sigma}=\lambda_{\sigma}>0$ on $S$.

We now introduce a semialgebraic map $r: V \rightarrow \bar{X}$ by the formula $(x \in V)$

$$
r(x)=\left(\sum_{\sigma} \mu_{\sigma}(x)\right)^{-1} \cdot\left(\sum_{\sigma} \mu_{\sigma}(x) \hat{\sigma}\right),
$$

with $\sigma$ running through $\Sigma(\bar{X})$.

Lemma 2.5. $r(S) \subset \bar{S} \cap A$ for every open simplex $S \subset V$ of $X^{\prime}$, and $r(x)=x$ for every $x \in A$.

Thus $r(V)=A$ and $] x, r(x)[\subset V$ for every $x \in V$. The map $r$ : $V \rightarrow A$ is a retraction as claimed in Proposition 2.2.

Proof of Lemma 2.5. Recall from the proof of the preceding lemma that $S=] \hat{\sigma}_{0}, \ldots, \hat{\sigma}_{n}\left[\right.$ with $\sigma_{0}<\cdots<\sigma_{n}, \sigma_{n} \subset X$, and $\sigma_{k} \subset A$ for some $k \in\{0, \ldots, n\}$. Let $t$ be the maximal index in $\{0, \ldots, n\}$ with $\sigma_{t} \subset A$. Consider some $\sigma \in \Sigma(\bar{X})$ with $\mu_{\sigma} \mid S \neq 0$. Then $\lambda_{\sigma} \mid S \neq 0$, hence $\sigma=\sigma_{k}$ for some $k \in\{0, \ldots, n\}$. For $k>t$ the function $\mu_{\sigma}$ would vanish on $S$. Thus $k \leq t$, and we obtain

$$
r(S) \subset] \hat{\sigma}_{0}, \ldots, \hat{\sigma}_{t}[\subset \bar{S} \cap A .
$$

If $S \subset A$ then $t=n$ and $\mu_{\sigma}=\lambda_{\sigma}$ on $S$ for every $\sigma \in \Sigma(\bar{X})$. Thus $r(x)=x$ for every $x \in A$. 
This finishes the proof of Proposition 2.2. We call the retraction $r$ : $V \rightarrow A$ defined above the canonical neighbourhood retraction $r_{X, A}$ of $A$ in $X$.

In order to prove Theorem 2.1 we need the explicit description of $\mathrm{St}_{X^{\prime}}(A)$ and its closure in terms of barycentric coordinates in the most simple case.

LEMMA 2.6. Let $\Delta=\left[e_{0}, \ldots, e_{n}\right]$ be a closed $n$-simplex in some space $R^{N}$, regarded as a geometric simplicial complex with its standard triangulation. Let $\sigma$ denote the open face $] e_{0}, \ldots, e_{r}[$ for some $r<n$. Then a point $x=\lambda_{0} e_{0}+\cdots+\lambda_{n} e_{n}$ of $\Delta\left(\right.$ all $\left.\lambda_{l} \geq 0, \lambda_{0}+\cdots+\lambda_{n}=1\right)$ lies in the star $\mathrm{St}_{\Delta^{\prime}}(\sigma)$ of $\sigma$ with respect to the first barycentric subdivision $\Delta^{\prime}$ if and only if

$$
\lambda_{m}<\min \left(\lambda_{0}, \ldots, \lambda_{r}\right) \text { for } r<m \leq n,
$$

and $x$ lies in the closure $\overline{\mathrm{St}}_{\Delta^{\prime}}(\sigma)$ of this star if and only if

$$
\lambda_{m} \leq \min \left(\lambda_{0}, \ldots, \lambda_{r}\right) \quad \text { for } r<m \leq n .
$$

Proof. We choose an element $\pi$ in the symmetric group $\delta(0, \ldots, n)$ such that

$$
\lambda_{\pi(0)} \geq \lambda_{\pi(1)} \geq \cdots \geq \lambda_{\pi(n)},
$$

and use the notations

$$
f_{l}:=e_{\pi(i)}, \quad \mu_{l}:=\lambda_{\pi(l)} \quad \text { for } 0 \leq i \leq n,
$$

and $\mu_{n+1}:=0$. Then

$$
x=\sum_{i=0}^{n} \mu_{i} f_{i}=\sum_{i=0}^{n}\left[\mu_{i}\left(f_{0}+\cdots+f_{l}\right)-\mu_{l+1}\left(f_{0}+\cdots+f_{i}\right)\right] .
$$

Introducing the simplices of $\Delta$,

$$
\left.\tau_{\iota}:=\right] f_{0}, \ldots, f_{i}[, \quad 0 \leq i \leq n,
$$

and their barycenters,

$$
\hat{\tau}_{i}=(i+1)^{-1}\left(f_{0}+\cdots+f_{l}\right),
$$

we have

$$
x=\sum_{i=0}^{n} \gamma_{l} \hat{\tau}_{l}, \quad \text { with } \gamma_{l}:=(i+1)\left(\mu_{i}-\mu_{i+1}\right) .
$$

Since $\gamma_{1} \geq 0, \gamma_{0}+\cdots+\gamma_{n}=1$, we see that $x \in\left[\hat{\tau}_{0}, \ldots, \hat{\tau}_{n}\right]$ and that $\gamma_{i}$ is the barycentric coordinate of $x$ corresponding to the vertex $\hat{\tau}_{i}$ of $\Delta^{\prime}$. Let

$$
j_{0}<j_{1}<\cdots<j_{s}
$$


denote those indices $i$ in $\{0,1, \ldots, n\}$ such that $\mu_{i}>\mu_{t+1}$. Then $] \hat{\tau}_{J_{0}}, \ldots, \hat{\tau}_{s_{s}}$ [ is the open simplex $T$ of $\Delta^{\prime}$ which contains $x$. Now $\bar{T} \cap \sigma \neq \varnothing$ if and only if $\hat{\sigma}$ is one of the vertices of $T$, and this means, since $\operatorname{dim} \sigma=r$, that $\sigma=\tau_{r}$ and $\mu_{r}>\mu_{r+1}$. Thus we see that $x$ lies in $\operatorname{St}_{\Delta^{\prime}}(\sigma)$ if and only if $\pi$ permutes the indices $0, \ldots, r$, i.e. $\pi \in \mathcal{S}(0, \ldots, r) \times \mathcal{S}(r+1, \ldots, n)$, and $\mu_{r}>\mu_{r+1}$. This is precisely condition (A) in the lemma. By an easy continuity argument one now verifies that $x$ lies in $\overline{\mathrm{St}}_{\Delta^{\prime}}(\sigma)$ if and only if condition (B) holds.

TheOREM 2.7. Again, let $X$ be a geometric simplicial complex over $R$ and $A$ be a closed subcomplex of $X$. Let $r: V \rightarrow A$ denote the canonical neighbourhood retraction of $A$ in $X, V:=\operatorname{St}_{X^{\prime}}(A)$, and let $U$ denote the star $\mathrm{St}_{X^{\prime \prime}}(A)$ of $A$ in the second barycentric subdivision $X^{\prime \prime}$ of $X$. The closure $\bar{U} \cap X$ of $U$ in $X$ is contained in $V$. For every $x \in \bar{U} \cap X$ the open line segment $] x, r(x)[$ is contained in $U$.

$N . B$. By this theorem

$$
H(x, t)=(1-t) x+\operatorname{tr}(x)
$$

is a strong deformation retraction from $\bar{U} \cap X$ to $A$ and also from $U$ to $A$. Thus Theorem 2.7 is a constructive version of Theorem 2.1 above.

Proof. Let $x \in \bar{U} \cap X$ be given. We choose an open simplex $\alpha=$ ]$\hat{S}_{0}, \ldots, \hat{S}_{k}$ [ of $X^{\prime \prime}$, which is contained in $U$ and which contains $x$ in its closure. Here $S_{0}<\cdots<S_{k}$ are simplices of $\bar{X}^{\prime}$, and $x$ is contained in an open face $] \hat{S}_{i_{0}}, \ldots, \hat{S}_{i_{r}}\left[\left(i_{0}<\cdots<i_{r}\right)\right.$ of $\alpha$. Thus $x \in S_{1}$ for some $l \in$ $\{0, \ldots, k\}$, namely $l=i_{r}$. Since $x \in X$, we have $S_{l} \subset X$. On the other hand, $S_{d} \subset A$ for some $d \in\{0, \ldots, k\}$, since $\bar{\alpha} \cap A \neq \varnothing$. Suppose that $d>l$. Then $S_{l}<S_{d}$, so $S_{l} \subset \bar{A} \cap X=A$. Thus we find an index $d \leq l$ with $S_{d} \subset A$. We see that $S_{l} \subset V$, and in particular $x \in V$.

We want to prove that $] x, r(x)\left[\subset U\right.$. We consider the closure of $S_{l}$,

$$
\Delta:=\bar{S}_{1}=\left[\hat{\sigma}_{0}, \ldots, \hat{\sigma}_{n}\right] \text {, }
$$

with open simplices $\sigma_{0}<\cdots<\sigma_{n}$ of $\bar{X}$. We regard $\Delta$ as a subcomplex of $\bar{X}^{\prime}$ and hence the first barycentric subdivision $\Delta^{\prime}$ as a subcomplex of $\bar{X}^{\prime \prime}$. Our point $x$ lies in the interior $\AA=S_{l}$ of $\Delta$. We have seen above that $x$ is contained in a face of $] \hat{S}_{0}, \ldots, \hat{S}_{l}[$. But the face $] \hat{S}_{0}, \ldots, \hat{S}_{d}[$ of $] \hat{S}_{0}, \ldots, \hat{S}_{l}[$ is contained in $A$. Thus $x \in \overline{\mathrm{St}}_{\Delta^{\prime}}(\Delta \cap A)$. We know from Proposition 2.2 that $] x, r(x)[\subset X$. Since

$$
\mathrm{St}_{\Delta^{\prime}}(\Delta \cap A) \cap X \subset \mathrm{St}_{X^{\prime \prime}}(A)
$$


the proof of the theorem will be finished as soon as we have verified

$$
] x, r(x)\left[\subset \mathrm{St}_{\Delta^{\prime}}(\Delta \cap A)\right. \text {. }
$$

This can be done by a lengthy but rather straightforward computation using Lemma 2.6. Let $\lambda_{0}, \ldots, \lambda_{n}$ denote the barycentric coordinates of $\Delta$ corresponding to the vertices $\hat{\sigma}_{0}, \ldots, \hat{\sigma}_{n}$. Let $t$ denote the maximal index in $\{0, \ldots, n\}$ with $\sigma_{t} \subset A$. If $t=n$, then $x \in \sigma_{n} \subset A$ and $x=r(x)$. Since nothing has to be proved in this case, we assume from now on $t<n$. The star $\mathrm{St}_{\Delta^{\prime}}(\Delta \cap A)$ is the union of the stars $\mathrm{St}_{\Delta^{\prime}}(T)$ with $T$ running through those faces of $] \hat{\sigma}_{0}, \ldots, \hat{\sigma}_{t}\left[\right.$ which lie in $A$. Thus $x \in \overline{\mathrm{St}}_{\Delta^{\prime}}(T)$ for one such face $T$. Let $\left\{\hat{\sigma}_{j} \mid j \in J\right\}$ denote the set of vertices of $T$. We have $J \subset$ $\{0, \ldots, t\}$ and, denoting the maximal index in $J$ by $m$, we have $\sigma_{m} \subset A$. By Lemma 2.6 we have for every $k \in\{0, \ldots, n\} \backslash J$,

$$
\lambda_{k}(x) \leq \min \left(\lambda_{J}(x) \mid j \in J\right) .
$$

Now consider a point

$$
y=(1-s) x+s r(x), \quad 0<s<1,
$$

in ] $x, r(x)$ [. The point $r(x)$ has the barycentric coordinates

$$
\lambda_{i}(r(x))= \begin{cases}0 & \text { if } t<i \leq n \\ \mu^{-1} w_{i} \lambda_{i}(x) & \text { if } 0 \leq i \leq t\end{cases}
$$

with $w_{i}=1$ if $\sigma_{i} \subset A$ and $\left.w_{i} \in\right] 0,1\left[\right.$ if $\sigma_{i} \not \subset A(0 \leq i \leq t)$, and some $\mu>0$. Thus

$$
\lambda_{i}(y)= \begin{cases}(1-s) \lambda_{l}(x), & t<i \leq n, \\ \left(1-s+w_{i} \mu^{-1} s\right) \lambda_{i}(x), & 0 \leq i \leq t .\end{cases}
$$

Let $J_{1}$ denote the set of all $k \in\{0, \ldots, n\}$ with

$$
\lambda_{k}(y) \geq \min \left(\lambda_{J}(y) \mid j \in J\right) .
$$

Of course, $J_{1} \supset J$. It is clear from $(*)$ that $J_{1} \subset\{0, \ldots, t\}$. We will verify that the open simplex $T_{1}$ spanned by the vertices $\hat{\sigma}_{j}, j \in J_{1}$, is contained in $A$. Then by Lemma 2.6

$$
y \in \mathrm{St}_{\Delta^{\prime}}\left(T_{1}\right) \subset \mathrm{St}_{\Delta^{\prime}}(\Delta \cap A)
$$

and our claim (?) is proved.

Let $l$ denote the maximal index in $J_{1}$. We have to show that $\sigma_{l} \subset A$. This certainly is true if $l=m(=\max J)$. Assume now that $l>m$, so $l \notin J$. We shall use the following simple observation, which is clear from 
the definition of the factors $w_{i}=w_{\sigma_{i}}(x)$. (Notice that a simplex $\sigma_{i}$ with $0 \leq i \leq t$ is contained in $X$ if and only if it is contained in $A$.) If $0 \leq i<j<k \leq t$ and $\sigma_{i}, \sigma_{k}$ are not contained in $A$, but $\sigma_{j} \subset A$, then $w_{i}>w_{k}$.

We obtain from $(* *)$ and $(*)$

$$
\begin{aligned}
\lambda_{l}(y) & =\left[(1-s)+\mu^{-1} w_{l} s\right] \lambda_{l}(x) \\
& \leq\left[(1-s)+\mu^{-1} w_{l} s\right] \min \left(\lambda_{j}(x) \mid j \in J\right),
\end{aligned}
$$

the inequality holding since $l \notin J$. Again by $(* *)$,

$$
\min \left(\lambda_{j}(y) \mid j \in J\right) \geq\left[(1-s)+\mu^{-1} \alpha s\right] \min \left(\lambda_{j}(x) \mid j \in J\right)
$$

with $\alpha=1$ or $\left.\alpha=w_{j} \in\right] 0,1\left[\right.$ for some $j \in J$ with $\sigma_{j} \not \subset A$. Now suppose that $\sigma_{l} \not \subset A$. If $\alpha=1$ then certainly $\alpha>w_{l}$. If $\alpha=w_{j}$ for some $j \in J$ such that $\sigma_{j} \not \subset A$, then $j<m<l$, and $\sigma_{m} \subset A$. Thus, by observation (***) again, $\alpha>w_{l}$. In both cases we obtain from the inequalities (1) and (2) that

$$
\lambda_{l}(y)<\min \left(\lambda_{j}(y) \mid j \in J\right)
$$

in contradiction to the fact that $l \in J_{1}$. Thus indeed $\sigma_{l} \subset A$. The proof of Theorem 2.7, as well as the proof of Theorem 2.1, is finished.

We mention two other nice properties of the canonical neighbourhood retraction $r_{X, A}$. Both these properties can be verified by calculations with barycentric coordinates in a straightforward manner.

REMARKS 2.8. Let $X$ be a geometric simplicial complex over $R$, let $A$ be a closed subcomplex of $X$, and let $r: \operatorname{St}_{X^{\prime}}(A) \rightarrow A$ denote the canonical neighbourhood retraction $r_{X, A}$. Further, let $\left.S=\right] \hat{\sigma}_{0}, \ldots, \hat{\sigma}_{n}[$ be an open simplex of $X^{\prime}$ contained in $\mathrm{St}_{X^{\prime}}(A)$ with $\sigma_{0}<\sigma_{1}<\cdots<\sigma_{n}$.

(i) For every $x \in S$ and $s \in R$ with $(1-s) x+s r(x) \in \bar{S} \cap \mathrm{St}_{X^{\prime}}(A)$ (e.g. $s \in[0,1])$ we have

$$
r((1-s) x+s r(x))=r(x) .
$$

(ii) $r(S)=] \hat{\sigma}_{0}, \ldots, \hat{\sigma}_{t}[$, where $t$ is the maximal index in $\{0, \ldots, n\}$ with $\sigma_{t} \subset A$.

$N$. B. Above we only verified that $r$ maps $S$ into $] \hat{\sigma}_{0}, \ldots, \hat{\sigma}_{t}[$. 
3. Locally complete spaces. All semialgebraic spaces are tacitly assumed to be separated.

DEFINITION 1. A semialgebraic space $M$ over $R$ is called locally complete if every point $x$ of $M$ has an affine semialgebraic neighbourhood $K_{x}$ which is a complete (cf. [DK $\left.\mathbf{D K}_{2}, \S 9\right]$ ) semialgebraic space.

Notice that then $x$ has a fundamental system of complete affine neighbourhoods. Indeed, for any semialgebraic embedding of $K_{x}$ into some $R^{n}$ the set $K_{x}$ is closed in $R^{n}$. Thus the intersection of $K_{x}$ with any closed euclidean ball in $R^{n}$ having $x$ as its center is a complete neighbourhood of $x$ in $M$.

EXAMPLE 3.1. If $M$ is a semialgebraic subset of $R^{n}$ which is locally closed in $R^{n}$, i.e. $M$ is open in its closure $\bar{M}$, then $M$ is locally complete.

EXAMPLE 3.2. For every algebraic variety $X$ over $R$ (separated and of finite type, as always) the space $X(R)$ of real points is locally complete. Indeed, choose for a given point $x$ of $X(R)$ a Zariski open affine neighbourhood $X_{1}$ of $x$ in $X$. Then $X_{1}(R)$ embeds into some $R^{n}$ as a closed semialgebraic subspace. Thus $x$ has a complete semialgebraic neighbourhood $L$ in $X_{1}(R)$. But $L$ is also a neighbourhood of $x$ in $X(R)$, and $L$ is affine.

We gather some elementary facts about locally complete spaces.

Proposition 3.3. Let $M$ be a locally complete semialgebraic subspace of $R^{n}$ for some $n>0$. Then $M$ is locally closed in $R^{n}$.

Proof. Let a point $x$ of $M$ be given. We have to show that for some $\varepsilon>0$ in $R$ the open euclidean ball $B_{\varepsilon}(x)$ of radius $\varepsilon$ with center $x$ does not meet the set $\bar{M} \backslash M$.

There exists some complete neighbourhood $L$ of $x$ in $M$. We choose $\varepsilon>0$ in such a way that $\bar{B}_{\varepsilon}(x) \cap M \subset L$, where $\bar{B}_{\varepsilon}(x)$ denotes the closed euclidean ball of radius $\varepsilon$ with center $x$. The semialgebraic set

$$
K:=\bar{B}_{\varepsilon}(x) \cap M=\bar{B}_{\varepsilon}(x) \cap L
$$

is closed in $L$, hence complete. Thus $K$ is closed in $R^{n}$. Clearly

$$
B_{\varepsilon}(x) \cap \bar{M} \subset \overline{B_{\varepsilon}(x) \cap M} \subset \bar{K}=K \subset M .
$$

Thus $B_{\varepsilon}(x)$ does not meet $\bar{M} \backslash M$. 
Proposition 3.4. Every affine locally complete space $M$ can be embedded into $R^{n}$ for some $n$ as a closed semialgebraic subspace.

Proof. (Cf. [Do, Chap. IV, 8.2].) We choose an embedding of $M$ into some space $R^{n}$. The set $\bar{M} \backslash M$ is semialgebraic and, by the preceding proposition, closed in $R^{m}$. Thus the function

$$
f: \bar{M} \rightarrow R, \quad f(x)=d(x, \bar{M} \backslash M),
$$

the distance from $x$ to $\bar{M} \backslash M$, is well defined and semialgebraic (according to Tarski) and takes only positive values on $M$. We embed $M$ into $R^{m+1}$ as the graph of $1 / f$,

$$
\alpha: M \rightarrow R^{m+1}, \quad \alpha(x):=\left(x, f(x)^{-1}\right) .
$$

Clearly $\alpha$ is a semialgebraic isomorphism from $M$ to $\alpha(M)$ and

$$
\alpha(M)=\{(x, t) \in \bar{M} \times R \mid f(x) t=1\}
$$

is closed in $R^{m+1}$.

Proposition 3.5. Every locally complete semialgebraic space $M$ is affine.

This can be deduced from the preceding Proposition 3.4 by a well known classical argument, cf. the proof of Proposition 8.8 in Chapter IV of Dold's book [Do]. It is evident that a locally complete semialgebraic space $M$ is regular. (Every open neighbourhood $U$ of a given point $x$ contains a closed neighbourhood.) Thus Proposition 3.5 is also a consequence of Robson's Theorem 1.3.

DEFinition 2. In analogy to classical terminology ([Do, p. 81]), we call a semialgebraic space $M$ an euclidean neighbourhood retract, if $M$ can be embedded into some $R^{n}$, i.e. $M$ is affine, and if for any such embedding $M$ is the retract of some semialgebraic neighbourhood $U$ in $R^{n}$.

As a consequence of the theory developed up to now we obtain

TheOREM 3.6. For an affine semialgebraic space $M$ the following statements are equivalent:

(a) $M$ is an euclidean neighbourhood retract.

(b) There exists an embedding of $M$ into some $R^{n}$ such that $M$ is retract of a semialgebraic neighbourhood $U$ in $R^{n}$.

(c) $M$ is locally complete. 
(d) For every embedding of $M$ into some affine semialgebraic space $N$ there exists a semialgebraic neighbourhood $U$ of $M$ in $N$, such that $M$ is a retract of $U$.

Proof. The implications (d) $\Rightarrow(\mathrm{a})$ and (a) $\Rightarrow(\mathrm{b})$ are trivial. (b) $\Rightarrow(\mathrm{c})$ : If $M$ is a semialgebraic subspace of $R^{n}$, and if there exists a neighbourhood $U$ of $M$ in $R^{n}$ such that $M$ is a retract of $U$, then $M$ is also a retract of the interior $\dot{U}^{\circ}$ of $U$. Thus $M$ is closed in $\dot{U}^{\circ}$, which implies that $M$ is locally complete. (c) $\Rightarrow(\mathrm{d})$ : By Proposition 3.3 there exists, for every embedding $M \hookrightarrow R^{n}$, an open semialgebraic subset $U \supset M$ of $R^{n}$ such that $M$ is closed in $U$. By Theorem 2.1 there exists an open semialgebraic subset $V$ of $U$ containing $M$ such that $M$ is a retract of $V$.

We return to the study of the canonical neighbourhood retractions, introduced in $\$ 2$, in the case of locally complete spaces. We need the following criterion for a semialgebraic map to be proper (cf. [DK $\left.\mathbf{D K}_{2}, \S 9\right]$ for the definition of proper maps). Notice the analogy of the criterion to a well known criterion for properness in topology [Bo, Chap. I, §10, No. 3].

Proposition 3.7. Let $f: M \rightarrow N$ be a semialgebraic map into a locally complete semialgebraic space $N$. The following are equivalent:

(a) $f$ is proper.

(b) For every complete semialgebraic subset $B$ of $N$ the preimage $f^{-1}(B)$ is again complete.

Proof. (a) $\Rightarrow(b)$ : This is the trivial implication. The restriction $f^{-1}(B) \rightarrow B$ of $f$ is proper, and $B$ is complete. Thus $f^{-1}(B)$ is complete.

(b) $\Rightarrow(\mathrm{a})$ : All the fibres of $f$ are complete. Thus, by $\left[\mathbf{D K} \mathbf{K}_{2}\right.$, Theorem 12.5], we may show that $f$ is proper by verifying that the image $f(A)$ of any closed semialgebraic subset $A$ of $M$ is closed in $N$. Let $y$ be a point in the closure $\overline{f(A)}$ of $f(A)$. Choose a complete neighbourhood $B$ of $y$ in $N$. The restriction $f^{-1}(B) \rightarrow B$ of $f$ is proper, since $f^{-1}(B)$ is complete by assumption. Thus $f\left(A \cap f^{-1}(B)\right)=f(A) \cap B$ is closed in $N$. In particular $y \in f(A) \cap B$, hence $y \in f(A)$.

We continue with the combinatorial situation and notations from $§ 2$. Let $X$ be a geometric simplicial complex over $R$ and $A$ a closed subcomplex. $V$ denotes the star $\mathrm{St}_{X^{\prime}}(A)$ of $A$ in the first barycentric subdivision $X^{\prime}$ of $X$, and $U$ denotes the star $\operatorname{St}_{X^{\prime \prime}}(A)$ in the second barycentric subdivision. Recall from Theorem 2.7 that $\bar{U} \cap X \subset V$. Let $r: V \rightarrow A$ denote the canonical neighbourhood retraction $r_{X, A}$ and let $H: V \times I \rightarrow V$ 
be the corresponding linear deformation retraction, $H(x, t)=(1-t) x+$ $\operatorname{tr}(x)$. Let $r_{1}: \bar{U} \cap X \rightarrow A$ and $H_{1}:(\bar{U} \cap X) \times I \rightarrow \bar{U} \cap X$ denote the restrictions of $r$ and $H$ to $\bar{U} \cap X$ and $(\bar{U} \cap X) \times I$ respectively.

THEOREM 3.8. Assume that $X$, regarded as a semialgebraic space, is locally complete. Then the maps $r_{1}$ and $H_{1}$ are proper.

Proof. We first prove the properness of $r_{1}$. It then will be an easy matter to see, that also $H_{1}$ is proper. By the preceding proposition, it suffices to verify that, for any given complete semialgebraic subset $B$ of $A$, the preimage $r_{1}^{-1}(B)=r^{-1}(B) \cap \bar{U}$ is again complete. Note that $r^{-1}(B) \cap \bar{U}$ is the union of the sets $r^{-1}(B) \cap \bar{U} \cap \bar{S}$ with $S$ running through the open simplices of $X^{\prime}$ in $V$. It suffices to verify the set $r^{-1}(B)$ $\cap \bar{U} \cap \bar{S}$ is complete for a given simplex $S$. Now $r(\bar{U} \cap \bar{S}) \subset \bar{S}$, thus

$$
r^{-1}(B) \cap \bar{U} \cap \bar{S}=r^{-1}(B \cap \bar{S}) \cap \bar{U} \cap \bar{S} .
$$

Replacing $B$ by $B \cap \bar{S}$, we may assume that $B \subset A \cap \bar{S}$.

We have to prove that $r^{-1}(B) \cap \bar{U} \cap \bar{S}$ is closed in $\bar{S}$. Let $y$ be a point in the closure of $r^{-1}(B) \cap \bar{U} \cap \bar{S}$. If $y \in X$ then $y \in X \cap \bar{U} \subset V$. Since $r$ is continuous, $r^{-1}(B)$ is closed in $V$. Consequently we have $y \in r^{-1}(B)$, hence $y \in r^{-1}(B) \cap \bar{U} \cap \bar{S}$. Thus our job is to prove that the closure of $r^{-1}(B) \cap \bar{U} \cap \bar{S}$ is disjoint from the subcomplex $Y:=\bar{X} \backslash X$ of $\bar{X}$. By Proposition 3.3 this subcomplex is closed in $\bar{X}$, since $X$ is locally complete. We will prove the following stronger claim:

$(*) r^{-1}(B) \cap \bar{S}$ has no adherence points in $Y$.

We have $S=] \hat{\sigma}_{0}, \ldots, \hat{\sigma}_{n}\left[\right.$ with open simplices $\sigma_{0}<\sigma_{1}<\cdots<\sigma_{n}$ of $\bar{X}$, and $\sigma_{n} \subset X$. Some of the $\sigma_{i}$ are contained in $A$. Let $t$ denote the maximal index $i$ with $\sigma_{i} \subset A$. Then $\sigma_{t} \not \subset Y$. Since the complex $Y$ is closed, we conclude that $\sigma_{i} \subset X$ for $t<i \leq n$. If $\sigma_{i} \subset X$ for every $i \in\{0, \ldots, n\}$, then $\bar{S} \cap Y=\varnothing$, and our claim $(*)$ is trivially true. From now on we assume that $\sigma_{i} \subset Y$ for some $i \in\{0, \ldots, n\}$, and we denote by $m$ the maximal index $i$ such that $\sigma_{i} \subset Y$. Since $Y$ is closed we have $\sigma_{i} \subset Y$ for $0 \leq i \leq m$. Since we know that $\sigma_{t} \subset A \subset X$, certainly $m<t$. For $m<i \leq t$ we have $\sigma_{i} \subset A$, since $A$ is closed in $X$.

Let $\lambda_{0}, \ldots, \lambda_{n}$ denote the barycentric coordinates on $\bar{S}$ corresponding to the vertices $\hat{\sigma}_{0}, \ldots, \hat{\sigma}_{n}$. The functions $w_{i}:=w_{\sigma_{i}}$, introduced in $\S 2$, take 
the following values on $X \cap \bar{S}: w_{i}(x)=0$ if $i>t, w_{l}(x)=1$ if $m<i \leq t$,

$$
w_{l}(x)=\left(\sum_{J=m+1}^{n} \lambda_{j}(x)\right)^{-1} \cdot\left(\sum_{j=m+1}^{t} \lambda_{j}(x)\right)
$$

if $0 \leq i \leq m$. In particular, $w_{i}=w_{0}$ for $0 \leq i \leq m$. For any $x \in V \cap \bar{S}$ the point $r(x)$ has, by definition of $r$, the barycentric coordinates

$$
\lambda_{i}(r(x))= \begin{cases}0 & t<i \leq n, \\ \mu(x)^{-1} \lambda_{i}(x) & m<i \leq t, \\ \mu(x)^{-1} w_{0}(x) \lambda_{l}(x), & 0 \leq i \leq m,\end{cases}
$$

with some nowhere vanishing function $\mu$ on $V \cap \bar{S}$. Clearly $Y \cap \bar{S}$ is the closed simplex $\left[\hat{\sigma}_{0}, \ldots, \hat{\sigma}_{m}\right]$. Thus $Y \cap \bar{S}$ is the set of zeros of the function $\sum_{j=m+1}^{n} \lambda_{j}$ on $\bar{S}$. Our given set $B \subset A \cap \bar{S}$ is disjoint from $Y$. Since $B$ is complete, there exists some $c>0$ in $R$ such that for every $q \in B$

$$
\left[\sum_{j=0}^{m} \lambda_{j}(q)\right] \cdot\left[\sum_{j=m+1}^{t} \lambda_{j}(q)\right]^{-1} \leq c .
$$

Applying this estimate to $q=r(x)$ for some $x \in r^{-1}(B) \cap \bar{S}$, we obtain

$$
\left[w_{0}(x) \cdot \sum_{j=0}^{m} \lambda_{j}(x)\right] \cdot\left[\sum_{j=m+1}^{t} \lambda_{j}(x)\right]^{-1} \leq c .
$$

Inserting the formula for $w_{0}(x)$ from above, we get

$$
\sum_{j=0}^{m} \lambda_{j}(x) \leq c \cdot \sum_{j=m+1}^{n} \lambda_{j}(x)
$$

for every $x \in r^{-1}(B) \cap \bar{S}$. Of course, this estimate remains true for $x$ in the closure of $r^{-1}(B) \cap \bar{S}$ : If such a point $x$ were to lie in $Y \cap \bar{S}$ then $\sum_{j=m+1}^{n} \lambda_{j}(x)=0$ and, by the estimate, we would also have $\sum_{j=0}^{m} \lambda_{j}(x)=$ 0 . But this is impossible since the sum of all barycentric coordinates of $x$ is 1 . Thus our claim (*) is proved, and $r_{1}$ is proper.

If $C$ is a complete semialgebraic subset of $\bar{U} \cap X$, then $H_{1}^{-1}(C)$ is a closed subset of $r_{1}^{-1}(r(C)) \times[0,1]$, since $r$ maps every line interval $[x, r(x)]$ to one point $r(x)$ (Remark 2.8.i). Thus $H_{1}^{-1}(C)$ is complete, and we see that also $H_{1}$ is proper. This completes the proof of Theorem 3.8.

For any semialgebraic map $f: M \rightarrow N$ the mapping cylinder $Z(f)$ is defined to be the quotient of the disjoint union $(M \times I) \bigsqcup N$ in the category of separated semialgebraic spaces with respect to the equivalence relation $(x, 1) \sim f(x)$ for all $x \in M$, provided this quotient exists. In our 
situation, let $f$ denote the restriction $\partial U \rightarrow A$ of $r$, with $\partial U=(\bar{U} \cap X) \backslash U$. We have a natural semialgebraic map

$$
p:(\partial U \times I) \sqcup A \rightarrow \bar{U} \cap X,
$$

defined by $p(a)=a$ for $a \in A$ and $p(x, t)=(1-t) x+t f(x)=H(x, t)$ for $(x, t) \in \partial U \times I$.

LEMMA 3.9. $p$ is surjective.

This will be proved below. Notice that the fibres of $p$ are the equivalence classes of the equivalent relation above $(M=\partial U, N=U)$. We now assume again that $X$ is locally complete. Then, by Theorem 3.8, the map $p$ is proper. Now every proper surjection is "identifying" in the category of separated semialgebraic spaces. Thus $p$ identifies $\bar{U} \cap X$ with the mapping cylinder of $f$, and we arrive at Theorem 2 in the introduction.

It can be shown that for every proper map $f: M \rightarrow N$ between locally complete semialgebraic spaces the mapping cylinder $Z(f)$ exists and is again locally complete. The proof is not difficult but would take us too far afield.

We still have to prove Lemma 3.9. Consider an open simplex $S=$ ]$\hat{\sigma}_{0}, \ldots, \hat{\sigma}_{n}\left[, \sigma_{0}<\cdots<\sigma_{n}\right.$, in $V$. Let $x$ be a point in $S \cap \bar{U}$. Then $r(x)$ is contained in an open face $S_{1}$ of $S$ (Remark 2.8.ii). We want to show that $x$ lies in the image of $p$. If $x \in A$ this is obvious. Assume now that $x \notin A$. Let $L$ be the line spanned by $x$ and $r(x)$. The intersection $L \cap \bar{S}$ is a closed line segment $[r(x), z]$ on $L$ which contains $x$. Let $T$ be the open face of $S$ with $z \in T$. Since $z$ is a boundary point of $S$ the face $T$ is different from $S$. Suppose that $z \in V$. Then $r(z)$ is defined, and the line segment $[z, r(z)]$ is contained in $\bar{T}$. But $r(z)=r(x)$ by Remark 2.8.i, hence $x \in[z, r(z)] \subset \bar{T}$, a contradiction. Thus $z \notin V$ and $L \cap \bar{S} \cap V=$ $[r(x), z[$. We have $z=(1-s) \cdot x+s \cdot r(x)$ with some $s \in R, s \neq 1$. The barycentric coordinate $\lambda_{n}(z)=(1-s) \lambda_{n}(x)$ of $z$ with respect to $\hat{\sigma}_{n}$ is not zero. Thus $z$ is contained in $\sigma_{n} \subset X$. Using again Remark 2.8.i we conclude that $L \cap \bar{S} \cap X \cap \bar{U}=[r(x), z] \cap \bar{U}$ is a closed line segment $[r(x), y]$, contained in $[r(x), z$ [, and $r(y)=r(x)$. Now $U$ is open in $V$, hence $U \cap[r(x), z[$ is open in $[r(x), z[$, and we see that $y \notin U$. Thus $y \in \partial U$. Clearly $x \in p(\{y\} \times[0,1])$. This completes the proof of Lemma 3.9.

REMARK 3.10. In this proof we did not use that $X$ is locally complete. Therefore, if $X$ is an arbitrary geometric simplicial complex and $A$ is a 
closed subcomplex of $X$, then using Remark 2.8.i, we can still state the following: The deformation retraction $H(x, t)=(1-t) x+\operatorname{tr}(x)$ yields by restriction a semialgebraic isomorphism

$$
\partial U \times[0,1[\stackrel{\sim}{\rightarrow}(\bar{U} \cap X) \backslash A .
$$

4. Extension of semialgebraic maps. We first state a rather obvious consequence of Theorem 2.1. Let $A$ be a closed semialgebraic subset of an affine space $M$, and let $U$ be a neighbourhood of $A$ in $M$ as described in Theorem 2.1. Let $I$ denote the unit interval $[0,1]$ of $R$.

Proposition 4.1. (Extension of semialgebraic maps to a neighbourhood.) Any semialgebraic map $f: A \rightarrow Z$ extends to a semialgebraic map $\tilde{f}$ : $\bar{U} \rightarrow Z$. If $\tilde{f}_{1}$ and $\tilde{f}_{2}$ are two extensions of $f$ to $\bar{U}$, then there exists a homotopy $F: \bar{U} \times I \rightarrow Z$ with $F_{0}=\tilde{f}_{1}, F_{1}=\tilde{f}_{2}$ and $F_{t} \mid A=$ f for every $t$ in $I$. (The same is true with $\bar{U}$ replaced by $U$.)

Indeed, let $H: \bar{U} \times I \rightarrow \bar{U}$ be a strong deformation retraction from $\bar{U}$ to $A$. Define $\tilde{f}:=f \circ H_{1}$, and define $F: \vec{U} \times I \rightarrow Z$ as follows:

$$
F(x, t)= \begin{cases}\tilde{f}_{1} \circ H(x, 2 t), & 0 \leq t \leq 1 / 2, \\ \tilde{f}_{2} \circ H(x, 2(1-t)), & 1 / 2 \leq t \leq 1 .\end{cases}
$$

We give two examples using the second statement in this proposition.

EXAMPLE 4.2. Let $A, M, U$ be as before, and let $\rho: \bar{U} \rightarrow A$ be any retraction from $\bar{U}$ to $A$. Applying the proposition with $Z=\bar{U}, f=\mathrm{id}_{\bar{U}}$, $g=i \circ \rho, i$ the inclusion map from $A$ to $\bar{U}$, we see that there exists a strong deformation retraction $\Phi: \bar{U} \times I \rightarrow \bar{U}$ with $\Phi_{1}=\rho$.

EXAMPLe 4.3. (Uniform local contractibility, cf. [Do, p. 81].) Let $M$ be any affine semialgebraic space. Applying the proposition to $M \times M$, the diagonal $\Delta$ of $M \times M$, and the canonical projections $p_{1}$ and $p_{2}$ from $M \times M$ to $M$, restricted to a suitable neighbourhood $L$ of $\Delta$ in $M \times M$, we see: There exists a semialgebraic closed neighbourhood $L$ of $\Delta$ in $M \times M$ and a semialgebraic map $H: L \times I \rightarrow M$ with the following properties:

(1) $H(x, x, t)=x$ for $x \in M, t \in[0,1]$,

(2) $H(x, y, 0)=x$ and $H(x, y, 1)=y$ for $(x, y) \in L$. 
We now ask for extension of a semialgebraic map $f: A \rightarrow Z$ to the whole of $M$.

Definition 1. (Cf. [S, p. 54].) We call a semialgebraic space $N$ solid, if for every pair $(M, A)$ consisting of an affine semialgebraic space $M$ and a semialgebraic closed subset $A$ of $M$ every semialgebraic map $f: A \rightarrow N$ extends to a semialgebraic map $\tilde{f}: M \rightarrow N$.

It would be bad to delete the word "affine" in this definition, since then not even the unit interval $[0,1]$ would be solid. (It is solid by our definition, cf. Theorem 4.5 below.)

REMARK 4.4. Every affine solid space $N$ is strongly contractible into every point $y_{0}$ of $N$, i.e. $\left\{y_{0}\right\}$ is a strong deformation retract of $N$.

For the proof, apply the definition to the pair

$$
(M, A):=\left(N \times I,(N \times\{0\}) \cup(N \times\{1\}) \cup\left(\left\{y_{0}\right\} \times I\right)\right)
$$

and the map $F: A \rightarrow N$ defined by

$$
F(y, 0)=y, \quad F(y, 1)=y_{0}, \quad F\left(y_{0}, t\right)=y_{0}
$$

$(y \in N, t \in I)$.

Definition 2. A semialgebraic space $N$ is called contractible if there exists a point $y_{0} \in N$ and a semialgebraic map $\Phi: N \times I \rightarrow N$ with $\Phi(y, 0)=y, \Phi(y, 1)=y_{0}$ for every $y \in N$. We call $\Phi$ a contraction of $N$ to $y_{0}$.

- TheOrem 4.5. Every contractible semialgebraic space $N$ is solid. Thus an affine space is solid if and only if it is contractible.

Proof. Let $M$ be an affine semialgebraic space, $A$ a closed semialgebraic subset of $M$ and $f: A \rightarrow N$ a semialgebraic map. We have to extend $f$ to a semialgebraic map $\tilde{f}: M \rightarrow N$. We choose an open semialgebraic neighbourhood $U$ of $A$ in $M$ with a retraction $r: \bar{U} \rightarrow A$. We further choose a semialgebraic function $\lambda: M \rightarrow[0,1]$ with $\lambda^{-1}(0)=A$ and $\lambda^{-1}(1)$ $=M-U$. This is possible by Theorem 1.6. Then we define the extension $f: M \rightarrow N$ of $f$ as follows:

$$
\tilde{f}(x)= \begin{cases}\Phi(f \circ r(x), \lambda(x)), & x \in \bar{U}, \\ y_{0}, & x \in M \backslash U,\end{cases}
$$

with $\Phi$ a contraction from $N$ to $y_{0}$. 
We mention a partial result on a refined version of our extension problem.

Corollary 4.6. Assume that $N$ is a semialgebraic space, $B$ is a semialgebraic subset of $N$, and that there exists a contraction $\Phi: N \times I \rightarrow N$ of $N$ into some point $y_{0} \in N \backslash B$ such that $\left.\Phi(N \times 10,1]\right) \subset N \backslash B$. Let $M$ be an affine semialgebraic space, $A$ a closed semialgebraic subset of $M$ and $f$ : $A \rightarrow B$ a semialgebraic map. Then there exists a semialgebraic map $\tilde{f}$ : $M \rightarrow N$ with $\tilde{f}^{-1}(B)=A$ and $\tilde{f} \mid A=f$.

Indeed, we obtain such a map $f: M \rightarrow N$ by the same formula as in the proof of Theorem 4.5.

In the special case $N=[0,1], B=\{0,1\}$ this corollary gives back our previous Theorem 1.6, which is thus put into a more general context.

5. Extension of homotopies. As before $I$ denotes the unit interval $[0,1]$ in $R$.

Definition. Let $M$ be a semialgebraic space and $A$ a semialgebraic subspace of $M$. We say that the pair $(M, A)$ has the homotopy extension property if the following holds: Given a semialgebraic map $g: M \rightarrow Z$ and a (semialgebraic) homotopy $F: A \times I \rightarrow Z$ into some semialgebraic space $Z$ with $F_{0}=g \mid A$, there exists a homotopy $G: M \times I \rightarrow Z$ such that $G_{0}=g$ and $G \mid A \times I=F$.

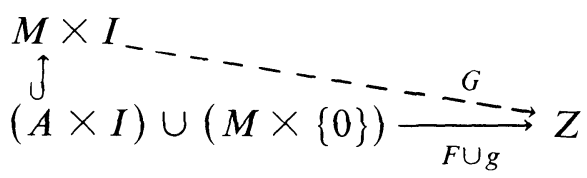

As in topology, it is evident that $(M, A)$ has the homotopy extension property if and only if $(A \times I) \cup(M \times\{0\})$ is a retract of $M \times I$. In this case $(A \times I) \cup(M \times\{0\})$ is closed in $M \times I$, and so $A$ is closed in M.

THEOREM 5.1. (Homotopy extension theorem.) If $M$ is an affine semialgebraic space and $A$ is a closed semialgebraic subset of $M$ then $(A \times I) \cup$ $(M \times\{0\})$ is a strong deformation retract of $M \times I$. In particular $(M, A)$ has the homotopy extension property.

Proof. We may assume that $M$ is a geometric simplicial complex in some $R^{n}$ and $A$ is a closed subcomplex of $M$. By Theorem 2.7 we have an 
open semialgebraic neighbourhood $U$ of $A$ in $M$ with a retraction $r$ : $\bar{U} \cap M \rightarrow A$, such that for every $x \in \bar{U} \cap M$ the line segment $[x, r(x)]$ is contained in $\bar{U} \cap M$ and for $x \in U$ this line segment is contained in $U$. We choose a semialgebraic function $f: M \rightarrow[0,1]$ with $f^{-1}(0)=M \backslash U$ and $f^{-1}(1)=A$. We introduce the following semialgebraic subsets $B, C, D, E$ of $M \times I \subset R^{n+1}$.

$$
\begin{aligned}
& B:=\left\{(x, t) \in(\bar{U} \cap M) \times I \mid \frac{1}{2} \leq f(x)<1,2(1-f(x)) \leq t \leq 1\right\}, \\
& C:=\left\{(x, t) \in(\bar{U} \cap M) \times I \mid \frac{1}{2} \leq f(x)<1,0 \leq t \leq 2(1-f(x))\right\}, \\
& D:=\left\{(x, t) \in(\bar{U} \cap M) \times I \mid 0 \leq f(x) \leq \frac{1}{2}\right\} \\
& E:=(M \backslash U) \times I .
\end{aligned}
$$

We have

$$
M \times I=(A \times I) \cup B \cup C \cup D \cup E .
$$

The sets $D$ and $E$ are closed in $M \times I$, while

$$
\bar{B} \cap(M \times I)=B \cup(A \times I), \quad \bar{C} \cap(M \times I)=C \cup(A \times\{0\}) .
$$

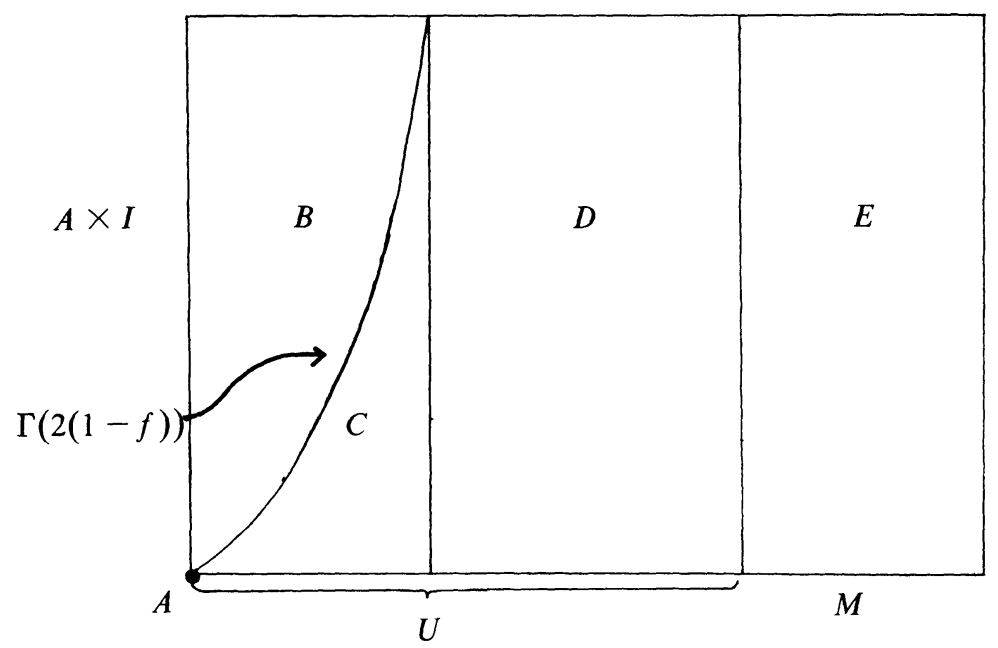

Picture of $M \times I$.

We shall use the following auxiliary maps:

$$
\begin{array}{ll}
g:(\bar{U} \cap M) \times I \rightarrow(\bar{U} \cap M) & g(x, t)=(1-t) x+\operatorname{tr}(x) \\
\varphi: C \rightarrow[0,1] & \varphi(x, t)=t / 2(1-f(x)) .
\end{array}
$$

We now define a map

$$
\rho: M \times I \rightarrow(A \times I) \cup(M \times\{0\})
$$

as follows, hoping that it is a retraction. 


$$
\begin{aligned}
& \rho(x, t):=(x, 0) \text { for }(x, t) \in E \\
& \rho(x, t):=(1-t) \cdot(x, 0)+t \cdot(g(x, 2 f(x)), 0) \text { for }(x, t) \in D \\
& \rho(x, t):=(1-\varphi(x, t)) \cdot(x, 0)+\varphi(x, t) \cdot(r(x), 0) \text { for }(x, t) \in C \\
& \rho(x, t):=(r(x), t-2(1-f(x))) \text { for }(x, t) \in(A \times I) \cup B .
\end{aligned}
$$

Notice that this map is well-defined, and takes values in

$$
(A \times I) \cup(M \times\{0\}),
$$

and is the identity on $(A \times I) \cup(M \times\{0\})$. Clearly $\rho$ has a semialgebraic graph. It remains to verify that $\rho$ is continuous. This is a problem only at the points of $A \times\{0\}$. Moreover, since $\rho$ is continuous on the closed subset $(A \times I) \cup B$ of $M \times I$, it suffices to check that for a given point $a$ of $A$ the values $\rho(x, t)$ converge to $(a, 0)$ if $(x, t) \in C$ and $(x, t)$ tends to $(a, 0)$. But this is clear, since

$$
\rho(x, t)-(a, 0)=(r(x)-a, 0)+(1-\varphi(x, t))(x-r(x), 0) .
$$

Thus $\rho$ is indeed a retraction. Now observe that for every $(x, t) \in M \times I$ the closed line segment $[(x, t), \rho(x, t)]$ is contained in $M \times I$, since, for $x \in \bar{U} \cap M$, the line segment $[x, r(x)]$ is contained in $M$. (It is even contained in $\bar{U} \cap M$.) The linear homotopy from id $\mathrm{i}_{M \times I}$ to $\rho$ is the desired strong deformation retraction from $M \times I$ onto $(A \times I) \cup(M \times\{0\})$.

Using Theorem 5.1, we can supplement Proposition 4.1 as follows.

Proposition 5.2. (Extension of homotopies into a neighbourhood.) Let $A$ be a closed semialgebraic subset of an affine semialgebraic space $M$, and let $U$ be a neighbourhood of $A$ in $M$ as described in Theorem 2.1. Let $f$ and $g$ be semialgebraic maps from $\vec{U}$ to a semialgebraic space $Z$, and let $F$ be $a$ homotopy from $f \mid A$ to $g \mid A$. Then there exists a homotopy $\tilde{F}$ from $f$ to $g$ which extends $F$. (The same is true with $\bar{U}$ replaced by $U$.)

Proof. By Theorem 5.1 there exists a homotopy $G: \bar{U} \times I \rightarrow Z$ with $G \mid A \times I=F$ and $G_{0}=f$. Put $G_{1}=h$. Then $h|A=g| A$. By Proposition 4.1 there exists a homotopy $H$ from $h$ to $g$ with $H_{t}|A=g| A$ for all $t \in I$. Composing $G$ and $H$ we obtain a homotopy $\hat{F}: \vec{U} \times I \rightarrow Z$ from $f$ to $g$. Let $B$ denote the closed subspace $(A \times I) \cup(\bar{U} \times\{0\}) \cup(\bar{U} \times\{1\})$ of $\bar{U} \times I$. We have an obvious homotopy $\Phi$ from the map $\hat{F} \mid B$ to the map $F \cup f \cup g$ on $B$. Using Theorem 5.1 again we extend $\Phi$ to a homotopy $\tilde{\Phi}$ on $\bar{U} \times I$ with $\tilde{\Phi}_{0}=\hat{F}$. Then $\tilde{F}:=\tilde{\Phi}_{1}$ is a map from $\bar{U} \times I$ to $Z$ which extends $F \cup f \cup g$, and hence is a homotopy from $f$ to $g$ extending $F$. 
The reader, who has followed us so far will have little doubt that it is possible to develop a full-fledged homotopy theory for affine semialgebraic spaces over an arbitrary real closed field. We hope to take up this subject in the near future.

\section{REFERENCES}

[Bo] N. Bourbaki, Topologie Générale, Hermann, Paris, 1971.

[B] G. W. Brumfiel, Partially ordered rings and semialgebraic geometry, London Math. Soc. Lecture Notes Series 37, Cambridge University Press 1979.

[D] H. Delfs, Kohomologie affiner semialgebraischer Räume, Dissertation, Regensburg 1980.

$\left[\mathrm{DK}_{1}\right]$ H. Delfs and M. Knebusch, Semialgebraic topology over a real closed field I: Paths and components in the set of rational points of an algebraic variety, Math. Z., 177 (1981), 107-129.

$\left[\mathrm{DK}_{2}\right] \_$Semialgebraic topology over a real closed field II: Basic theory of semialgebraic spaces, Math. Z., 178 (1981), 175-213.

$\left[\mathrm{DK}_{3}\right] \ldots$, On the homology of algebraic varieties over real closed fields, J. Reine Angew. Math., 335 (1982), 122-163.

[Do] A. Dold, Lectures on Algebraic Topology, Springer, Berlin - Heidelberg - New York, 1972.

[R] R. Robson, Embedding semi-algebraic spaces, Math. Z., 183 (1983), 365-370.

[S] N. Steenrod, The Topology of Fibre Bundles, Princeton University Press, 1951.

Received August 2, 1982.

FAKULtät FUR MATHEMATIK DER UNIVERSität

D-8400 REGENSBURG-F.R.G. 



\section{PACIFIC JOURNAL OF MATHEMATICS \\ EDITORS}

DONALD BABBITt (Managing Editor)

University of California

Los Angeles, CA 90024

J. Dugundu

University of Southern California

Los Angeles, CA 90089-1113

R. FINN

Stanford University

Stanford, CA 94305

HERMANN FLASCHKA

University of Arizona

Tucson, AZ 85721
C. C. MOORE

University of California

Berkeley, CA 94720

ARTHur Ogus

University of California

Berkeley, CA 94720

Hugo RossI

University of Utah

Salt Lake City, UT 84112

H. SAMELSON

Stanford University

Stanford, CA 94305

ASSOCIATE EDITORS

R. ARENS

E. F. BECKENBACH

B. H. NeumanN (1906-1982)

F. WOLF

K. YoshidA

\section{SUPPORTING INSTITUTIONS}

UNIVERSITY OF ARIZONA

UNIVERSITY OF BRITISH COLUMBIA

UNIVERSITY OF OREGON

CALIFORNIA INSTITUTE OF TECHNOLOGY

UNIVERSITY OF CALIFORNIA

MONTANA STATE UNIVERSITY

UNIVERSITY OF SOUTHERN CALIFORNIA

UNIVERSITY OF NEVADA, RENO

STANFORD UNIVERSITY

UNIVERSITY OF HAWAII

NEW MEXICO STATE UNIVERSITY

UNIVERSITY OF TOKYO

UNIVERSITY OF UTAH

OREGON STATE UNIVERSITY

WASHINGTON STATE UNIVERSITY

UNIVERSITY OF WASHINGTON 


\section{Pacific Journal of Mathematics}

Vol. 114, No. $1 \quad$ May, 1984

David Marion Arnold and Charles Irvin Vinsonhaler, Typesets and cotypesets of rank-2 torsion free abelian groups $\ldots \ldots \ldots \ldots \ldots \ldots \ldots 1$

Duncan Alan Buell and Richard Howard Hudson, Solutions of certain

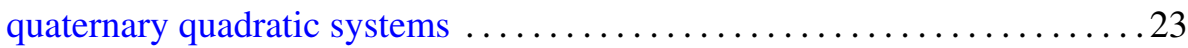

Hans Delfs and Manfred Knebusch, Separation, retractions and homotopy

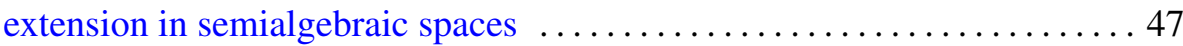

I. Erdélyi and Sheng-Wang Wang, A spectral duality theorem for closed

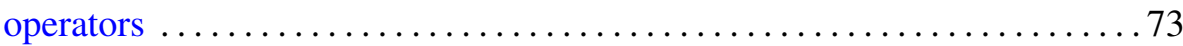

Theodore William Gamelin, Weak compactness of representing measures for $R(K)$

Kenneth R. Goodearl and T. H. Lenagan, Krull dimension of

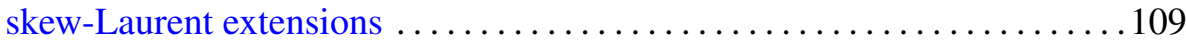

Daniel Joseph Gross, Compact quotients by $\mathbf{C}^{*}$-actions . . . . . . . . . . . 149

Goo Ishikawa, Satoshi Koike and Masahiro Shiota, Critical value sets of

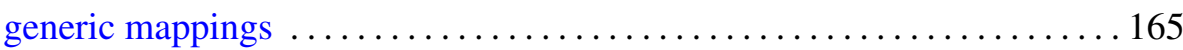

Hong Oh Kim, Derivatives of Blaschke products $\ldots \ldots \ldots \ldots \ldots \ldots \ldots \ldots \ldots$

Erhard Luft and Denis Karmen Sjerve, 3-manifolds with subgroups

$Z \oplus Z \oplus Z$ in their fundamental groups $\ldots \ldots \ldots \ldots \ldots \ldots \ldots \ldots \ldots \ldots \ldots \ldots$

George Clifford Nelson, Boolean powers, recursive models, and the Horn

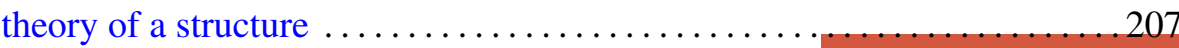

W. J. Phillips, Flow under a function and discrete decomposition of properly infinite $W^{*}$-algebras

Teodor C. Przymusiński, A solution to a problem of E. Michael ... 235

Bruce Harvey Wagner, Derivations of quasitriangular algebras 243 